\title{
¿Qué y cómo evaluar el desempeño docente? Una propuesta basada en los factores que favorecen el aprendizaje
}

\section{What and How to Assess Teacher's Performance? A Proposal Based on the Factors that Support Learning}

\author{
Jesús Carlos-Guzmán
}

Universidad Nacional Autónoma de México, Ciudad de México, México.

Doctor en Pedagogía. Maestro en Psicología Educativa. Coordinador de la Maestría para la Enseñanza Media Superior en el campo del conocimiento de psicología.

Recibido: 21-01-16

Aprobado: 02-05-16

\section{Correspondencia}

Email: jcarlosguzman@mac.com
Citar como:

Carlos-Guzmán, J. (2016). ¿Qué y cómo evaluar el desempeño docente? Una propuesta basada en los factores que favorecen el aprendizaje. Propósitos y Representaciones, 4(2), 285-358. Doi: http:// dx.doi.org/10.20511/pyr2016.v4n2.124

(C) Universidad San Ignacio de Loyola, Vicerrectorado de Investigación y Desarrollo, 2016. (e) BY.NC-ND Este artículo se distribuye bajo licencia CC BY-NC-ND 4.0 Internacional (http://creativecommons.org/licenses/by-nc-nd/4.0/). 


\section{Resumen}

Se presenta una propuesta para evaluar el desempeño docente basada en los aspectos que la investigación educativa ha encontrado como fundamentales para lograr el aprendizaje de los alumnos. La propuesta está dirigida principalmente a los docentes y responsables de programas de evaluación docente. Luego de criticar la manera tradicional de valorar el desempeño docente, se muestra una forma diferente para realizarlo considerando cuatro dimensiones esenciales de la enseñanza: Dominio de lo enseñado, manejo de los aspectos didácticos, el pensamiento didáctico del maestro y la motivación, responsabilidad y autoeficacia docente. Se describe y justifica el qué evaluar y el cómo hacerlo, lo cual se sugiere hacerlo mediante la observación de la práctica docente; por ello se dan lineamientos para valorarla, resaltando los aspectos didácticos por considerarlos cruciales para que los alumnos adquieran el conocimiento. Se termina resaltando sus ventajas y algunas sugerencias para poder aplicarla.

Palabras clave: Evaluación docente, factores que promueven el aprendizaje, didáctica, enseñanza efectiva.

\section{Summary}

A proposal is presented to assess teacher's performance based on the aspects that educational research has found as fundamental to achieve students' learning. The proposal is aimed mainly at teachers and people responsible for teacher's assessment programs. After criticizing the traditional way of assessing teacher's performance, it is shown a different way of doing it considering four essential dimensions of teaching: Knowledge of what is taught, management of didactic aspects, teacher's didactic thinking and teacher motivation, responsibility and self-efficacy. It is described and justified what and how to assess, suggesting to do it by observing the educational practice. Therefore, guidelines are provided to assess it, pointing out the didactic aspects that are considered essential for students 
to acquire knowledge. It ends pointing out its advantages and giving some recommendations to apply it.

Keywords: Teachers' performance, factors that promote learning, didactics, effective teaching. 


\section{Introducción}

Si el fin de la enseñanza es lograr el aprendizaje de los alumnos y alcanzar las metas establecidas de la enseñanza (Cross, 1993, como se citó en Huba \& Freed, 2000; Echeverría, 2011; Hativa, 2000), entonces la evaluación del docente debería valorar si las acciones del maestro contribuyen o no a obtener tal propósito.

Evaluar la labor docente de manera justa, integral y con propósitos formativos -es decir, ayudarlo a enseñar mejor y no únicamente valorarlo como un requisito a cumplir- ha significado un importante reto para la investigación educativa y para los responsables de aplicar estos programas en los diferentes países (Rueda, 2001; UNESCO, 2014).

La manera común de hacerlo ha sido por medio de cuestionarios aplicados a los estudiantes (Cisneros, Jorquera \& Aguilar, 2011; Rueda, 2001). Igualmente, se ha usado el portafolio (Cordero, 2002), los estudios de casos (Martínez Rizo, 2011), o juzgarlo a partir de los resultados de los aprendizajes o el rendimiento académico de sus alumnos en pruebas o exámenes masivos (Calderón \& De Oliveira, 2013); o por el denominado valor agregado, que compara el rendimiento escolar en dos momentos: al inicio y al final de la experiencia instruccional (OCDE, 2011). Todas estas formas tienen ventajas y limitaciones (Martínez Rizo, 2011), pero existe coincidencia en que el desempeño docente debe valorarse utilizando diferentes herramientas (Schmelkes, 2014; Jornet, González \& Bakieva, 2012) y no solo de una manera, como usualmente se hace.

Consideramos que la finalidad de la evaluación docente debe ser la mejora de la enseñanza y no solo juzgar al maestro; por ello, la propuesta presentada integra los resultados que la investigación educativa ha encontrado que inciden favorablemente en el aprendizaje de los estudiantes (Hativa, 2000; Hattie, 2009, 2012 y Schmelkes, 2014), así como las sugerencias desprendidas del estudio de las buenas prácticas de enseñanza (Bain, 2004, CarlosGuzmán, 2006, 2014). Esta dirigida sobre todo a los profesores de diferentes 
niveles educativos interesados en enseñar de mejor manera e, igualmente, para los estudiosos y responsables de diseñar y aplicar programas de evaluación docente.

Aclaramos que no pretendemos hacer una revisión sobre el estado del arte de la evaluación docente, sino ofrecer una alternativa a la manera tradicional de valorar al maestro. Tampoco pretendemos realizar un análisis epistemológico sobre este tema, sino integrar lo que sabemos sobre las mejores formas en que las personas aprenden y utilizar este conocimiento como pauta para considerarlo en la evaluación del docente. Es una propuesta genérica que puede emplearse con profesores de diferentes niveles educativos -haciendo las adecuaciones necesarias-, como más adelante se explica.

Partimos del hecho de que para evaluar al maestro hay que responder dos preguntas: una es seleccionar qué evaluar de la práctica docente, dada la amplia gama de factores que intervienen en ella, y la otra es cómo hacerlo, o sea, definir qué tipo de instrumentos son los adecuados para aprehender su complejidad. Para responder la primera pregunta nos basaremos en las actividades identificadas como cruciales por las investigaciones sobre los factores que promueven el aprendizaje y mejoran el rendimiento académico, incluyendo lo encontrado sobre las buenas prácticas de enseñanza (Bain, 2004; Onrubia y Rochera \& Colomina, 2001; Carlos-Guzmán, 2006, 2014; Hattie, 2009, 2012; Ramsden, 2007; Schmelkes, 2014). A partir de ahí identificamos diversas dimensiones, las mismas que sugerimos sean evaluadas empleando distintos instrumentos.

Así, este artículo está organizado en dos partes: en la primera fundamentamos lo propuesto y en la segunda lo describimos. Debido a que consideramos a la docencia un desempeño, es mejor valorarla observando la práctica docente dentro del aula, y no hacerlo mediante cuestionarios por considerarlos limitados para este fin, como más adelante expondremos.

En síntesis, nuestra propuesta toma en cuenta las dimensiones que, consistentemente, han demostrado que repercuten de manera favorable en 
la adquisición de los conocimientos escolares por parte de los alumnos. Empezaremos definiendo lo que entendemos por enseñar.

\section{¿Qué es enseñar?}

Enseñar es aquella actividad interconectada, propositiva, planeada y sistemática que, de forma deliberada, busca el aprendizaje de los educandos. Es un acto coordinado y dirigido por el maestro, dado que sin su concurso será difícil alcanzar las metas de enseñanza. Así lo estableció Dewey (2006), para quien educar significa crear un ambiente donde, utilizando determinados métodos y materiales, se promueve el desarrollo del educando en cierta dirección y, debido a su trascendencia, no se deja al azar, sino que se realiza propositivamente. Por ello, la enseñanza, de acuerdo con Zabala (2000), está compuesta por tres fases: planeación, ejecución y evaluación. Para llevarla a cabo se toma en cuenta al sujeto (quién aprende), el contenido (qué se aprende), así como sus fines.

La enseñanza es un proceso sistemático porque se realiza siguiendo una metodología compuesta por estrategias, métodos y técnicas didácticas que se aplican de manera secuencial, pertinente y organizada; para lograrlo, emplea unos medios (material didáctico, libros, TIC) y ocurre en un contexto que puede favorecerla o entorpecerla. El docente organiza los tiempos y las tareas, creando una atmósfera que estimule el aprendizaje. Su finalidad es alcanzar la comprensión del conocimiento y cumplir con metas, tanto de corto plazo (dominio de los conocimientos concretos de un grado o asignatura) como de largo plazo (vgr. contribuir a la formación integral del aprendiz o dominar las competencias para la vida). Es, también, una actividad ética que busca modificar a las personas en un sentido deseable, tanto para sí mismas como para la sociedad de la que forman parte. Los resultados de la enseñanza son comúnmente evaluados, y lo ahí encontrado debe contribuir a su mejora.

Enseñar, por lo tanto, implica que el docente es responsable de preparar las condiciones para que ocurra el aprendizaje y, a la vez, ayudar al estudiante a aprehenderlo. Meirieu (2005) lo resume así: "Enseñar es 
organizar la confrontación con el saber y proporcionar las ayudas para hacerlo propio" (p. 24).

La docencia es más que la aplicación de una serie de acciones (Saroyan, 2001), ya que es afectada por tipos particulares de pensamiento, creencias, sentimientos y actitudes del docente (Carlos-Guzmán, 2006; Goodyear \& Hativa, 2002; Monroy \& Díaz, 2001; Van den Berg, 2002) y por la forma como se integran los distintos saberes y conocimientos involucrados: disciplinarios, didácticos, contextuales, experienciales, etc.

\section{Críticas a la forma usual de valorar la docencia.}

Aceptar lo anterior implica que las maneras de evaluación de la docencia deben tomar en cuenta esta complejidad, dinamismo, diversidad y especificidad, lo cual usualmente no se hace. Como decíamos, el cuestionario ha sido la forma generalizada de evaluarla. Si bien tiene ventajas (García \& Medécigo, 2014) en el sentido de que, si está diseñado correctamente y cumple con los criterios psicométricos, recopila la opinión de los receptores de la labor docente como son los alumnos, puede aplicarse masivamente y cuantificar sus resultados; no obstante, muestra las siguientes desventajas (Arbesú \& Argumedo, 2010; Cisneros, Jorquera \& Aguilar, 2011; Patrón \& Cisneros, 2013).

\section{Es de amplio espectro.}

Al momento de diseñar un cuestionario de evaluación docente, existe la tendencia a considerar una gran cantidad de aspectos de su labor, entre los que destacan: si entrega y cumple con un programa académico, su metodología didáctica, sus actitudes hacia la docencia y los estudiantes, la forma de utilizar los materiales de apoyo, su ética, liderazgo, sus formas de evaluación, el grado de satisfacción de los estudiantes con su enseñanza, sin olvidar su puntualidad y asistencia. Como resultado, los cuestionarios son abultados en cuanto a sus reactivos, a veces contienen decenas de preguntas. Así se recaba mucha información, pero no queda clara la utilidad de la misma en cuanto a si todos los factores medidos afectan positivamente el aprendizaje 
de los alumnos. Sucede lo mismo en los procesos de acreditación; también se recopila gran cantidad de información sobre los aspectos administrativos y académicos de las instituciones, pero solo una mínima parte de ellos son variables importantes del rendimiento escolar de los aprendices (Loera y Cazares, 2005). Si lo relevante es centrarnos en los aspectos cruciales para el aprovechamiento académico del estudiante, entonces no tiene mucho sentido atiborrarnos de datos e información que, tal vez, sea innecesaria para este fin.

\section{Visión idealizada del maestro.}

Los cuestionarios reflejan, asimismo, una postura sobre la docencia en el sentido de querer valorar el deber ser y al profesor ideal. Por eso, es común encontrar que al docente se le sobrecarga de responsabilidades y deberes; algunos de ellos escapan a lo que estarían estrictamente obligados a cumplir, confundiendo lo deseable con lo exigible. Así, al maestro se le demanda que sea un líder académico, una persona culta, un promotor de valores, un modelo a seguir, un activo participante en su comunidad, un luchador social, un investigador, tener responsabilidad social, trabajar en colegiado, diseñar materiales, un terapeuta para atender los problemas psicológicos de los estudiantes $\mathrm{y}$, a veces, también de los padres de familia, un agente preventivo del acoso escolar, un gestor para obtener materiales y apoyos para la escuela, un administrador de los recursos escolares, un experto en las TIC, innovador, etc. ¿De verdad creemos que una persona puede ser capaz de mostrar todas estas características de manera eficiente? Si bien es deseable que el maestro tenga buena parte de estos rasgos, no creemos que sea posible, realista ni justo evaluar que reúna todos estos atributos. Así, puestos a elegir, y para seleccionar solo lo crucial para favorecer el aprendizaje de los estudiantes, es que debemos exigir al maestro que sea un profesional de la docencia, un experto en los procesos que favorecen el aprendizaje de sus alumnos y que actúe con sentido ético. De esta manera, la evaluación debería estar dirigida a valorar si el docente realiza las acciones que sabemos ayudan a la adquisición plena de los contenidos escolares. Esto es lo central; lo demás es secundario, aunque deseable. 


\section{Refleja una mirada simplista de la docencia.}

Los reactivos de los cuestionarios de evaluación docente generalmente son lineales, rígidos, en el sentido de concebir la práctica docente como un conjunto de acciones secuenciales que se manifiestan solo de una manera y, algunas de ellas, binarias al valorar solo la presencia o ausencia del atributo, no el grado en que se presenta. Pero, tal como describimos al inicio de este apartado, la docencia es una actividad compleja que escapa a esta visión simplista para evaluarla. Enseñar es un claro ejemplo de un procedimiento heurístico, donde ante una situación didáctica concreta hay varias opciones a considerar. Es decir, existen diferentes formas de responder ante la misma circunstancia, así como distintas acciones para lograr la meta de que los alumnos aprendan.

Por eso, la evaluación docente debe tomar en cuenta estos rasgos, pero generalmente no sucede así. También, como lo señala Díaz Barriga (2001), sus reactivos reflejan, habitualmente, un modelo transmisivo de docencia, y no uno activo o centrado en el alumno. Por eso, Andrerucci (2011) afirma que se corre un peligro cuando se simplifica y desnaturaliza la complejidad de la docencia.

\section{Excesivamente generalista.}

Otra importante limitación ha sido la de formular preguntas generalistas y descontextualizadas, asumiendo que todos los maestros deberían de hacerlas independientemente del tipo de materia que enseñan. Así, por ejemplo, se evalúa si el docente hace uso de las TIC o aplica el aprendizaje cooperativo sin tomar en cuenta si esas actividades son pertinentes para las características del curso, para el tipo de alumnos o, incluso, para el momento en que se encuentra el curso. Es decir, el uso de estas técnicas y estrategias no es adecuado o inapropiado per se, sino que su valor depende de diversas circunstancias, contenidos y momentos. Por eso, no debe valorarse calificando como presencia o ausencia, sino hacerlo de manera contextual; por lo tanto, 
determinarse si esas acciones son adecuadas o inadecuadas para el momento de la enseñanza evaluada. O sea, deberían juzgarse las interrelaciones y adecuaciones de las acciones, y no solo si se hacen o no.

En suma, se debe evaluar la docencia asumiendo su complejidad, grado de idoneidad y por la pertinencia de las acciones realizadas. Si bien una de las ventajas de los cuestionarios de evaluación docente es que se aplican masivamente, es también su limitación, ya que no toma en cuenta la amplia gama de formas de enseñar, usa categorías estáticas que no consideran las especificidades propias de cada disciplina, grado o nivel y lo que cada una de ellas valora o aprecia, que es diferente una de otra. Tampoco es sensible a las diferencias individuales ni ofrece información que pueda servir de diagnóstico (Saroyan, 2001).

Una vez señaladas las limitaciones a la manera usual de valorar la enseñanza, pasaremos a analizar las dimensiones que la investigación educativa ha encontrado como centrales de la práctica docente; las describiremos justificando su papel e importancia. Ellas fundamentan la propuesta que hacemos.

\section{Las dimensiones de la práctica docente.}

\section{Dominio de lo enseñado.}

Este aspecto parece muy obvio, pero no por eso hay que dejar de mencionarlo: un docente debe ser experto en el tema, asignatura o grado que imparte (Darling-Hammond, 2000); es un requisito indispensable manejar adecuadamente los contenidos escolares que enseña, sean estos del nivel de educación superior o de educación básica.

¿Esto qué implica? La necesidad de demostrar que comprende plenamente los hechos, conceptos y principios de la materia enseñada (Darling-Hammond \& Youngs, 2002).

Abarca también el dominio de las mejores formas para organizar y conectar las ideas, la capacidad para identificar los conceptos cruciales de 
su disciplina y diferenciarlos de los secundarios (Shulman, 1986 citado en Hativa, 2000). Algunos incorporan en este dominio saber diseñar programas académicos e integrar su materia dentro del enramado curricular para vincularlo con otras asignaturas, tanto vertical como horizontalmente (Posner, 2005).

El dominio disciplinar fue destacado también como importante por Hernández (1995). Sin embargo, es una condición indispensable pero no suficiente para la práctica docente, ya que el maestro puede ser un excelente experto en su tema, pero esto no lo habilita para saber enseñarlo, ya que puede incurrir en lo denominado por Nathan y Petrosino (2003) como ceguera disciplinaría, que es cuando dan un excesivo peso a los factores disciplinarios, descuidando lo didáctico o el cómo enseñarlo, dimensión que analizaremos a continuación.

\section{Manejo de los aspectos didácticos y de gestión de la clase.}

Este dominio lo dividiremos en dos: el primero, para referirnos a la parte didáctica o el manejo que hace el docente para hacer accesible y comprensible el contenido por enseñar; el segundo es la denominada gestión de la clase, es decir, el uso de técnicas para organizar, dirigir y coordinar la clase, e implica la capacidad para crear una atmósfera propicia para el aprendizaje (Gauthier, 1997).

\section{Dominio didáctico.}

Desde inicios del siglo XX, según lo reporta Butsch (1931), el saber enseñar o la capacidad didáctica es el rasgo que constantemente ha sido identificado como crucial de la práctica docente, tanto por alumnos de diferentes niveles educativos como por los directores escolares. ¿Qué rasgos comprende esta capacidad? Describiremos las principales cualidades del docente identificadas en los resultados de las investigaciones. 
Hattie (2009), luego de analizar 800 metaanálisis de estudios sobre los factores que afectan el rendimiento académico del estudiante, que implicó el análisis de miles de investigaciones y la participación de un poco más de un millón de alumnos, destaca seis aspectos importantes relacionados con la calidad docente:

a) Los maestros son una de las influencias más poderosas para lograr el aprendizaje.

b) Ellos necesitan ser directivos, influyentes, cuidadosos y apasionados por la enseñanza y el aprendizaje.

c) Los docentes requieren estar conscientes de la manera como cada alumno piensa y conoce, para diseñar experiencias significativas y dar una retroalimentación adecuada para ayudar a cada uno a avanzar progresivamente en los diferentes niveles curriculares.

d) Precisan especificar claramente las metas de enseñanza y los criterios de logro, para que todos sepan si, efectivamente, fueron alcanzados o no por los estudiantes.

e) Requieren identificar los procesos de construcción y reconstrucción del conocimiento de sus alumnos para que dominen los principios de aprendizaje involucrados en los contenidos enseñados, sabiendo que lo importante no es la adquisición del contenido como tal, sino los procesos cognoscitivos (razonamiento, solución de problemas, creatividad, pensamiento crítico, etc.) desarrollados al adquirir tal contenido.

f) Los directivos y los maestros necesitan crear ambientes de aprendizaje donde el error y las concepciones erróneas sean bienvenidas y vistas como oportunidades para el aprendizaje, en los cuales el aprendiz se sienta seguro para aprender, reaprender y explorar el conocimiento.

Ahora, por lo que toca al acto de enseñar, uno de los factores cruciales para que los estudiantes comprendan el contenido enseñado es la claridad, 
definida como la capacidad del docente para comunicar las ideas de manera clara y convincente (Assaél y Pavez, 2008; Darling-Hammond, 2000). Ella es imprescindible para lograr que los alumnos tengan una adecuada comprensión del contenido impartido (Hativa, 2000). Involucra la habilidad para presentar el contenido de forma lógica y organizada, utilizar ejemplos, explicar el tema de manera simple y amena, enseñar paso a paso, adaptar el contenido enseñado a las características y nivel de conocimiento de los estudiantes, responder adecuadamente a sus preguntas, explicar de otra manera cuando no han comprendido, enfatizar o destacar los puntos importantes del contenido para distinguir lo crucial de lo secundario, contrastar diferentes posturas, verificar el grado de comprensión del contenido por parte de los estudiantes y estimular su interés e involucramiento (Hativa, 2000). El profesor, además, debe usar adecuadamente la retroalimentación para corregir las realizaciones de los estudiantes al tener una importante repercusión positiva en su aprendizaje (Hattie, 2012; Shute, 2008). Así como utilizar el barómetro, es decir, verificar el comportamiento verbal y no verbal de los estudiantes y, a partir de ello, hacer cambios inmediatos a su docencia (Hativa, 2000).

Otras de las cualidades docentes identificadas son las de establecer y comunicar sus expectativas y lo que desea que aprendan sus alumnos, fomentar la autodisciplina, manejar adecuadamente el tiempo y los recursos de la clase, evaluar la comprensión de lo enseñado, asignar tareas vinculadas con los temas revisados en el aula (Mcber, 2000). También, despertar el interés planteando retos intelectuales (Ramsden, 2007) y estimulando el deseo de aprender por parte de los estudiantes, donde ellos acepten el esfuerzo que va a requerir. Finalmente, es importante presentar tareas que propicien la activación de los procesos cognoscitivos complejos y que no sean rutinarias, repetitivas, elementales o memorísticas (Hattie, 2012).

Es decir, un buen docente tiene como principal preocupación lograr el aprendizaje de sus alumnos, confrontarlos con retos alcanzables dándoles el apoyo necesario para resolverlos, confiar en sus capacidades creyéndolos personas que quieren aprender (Ramsden, 2007). Igualmente, conformar 
un clima propicio para el aprendizaje, despertar la motivación y el interés de los alumnos, aplicar formas sistematizadas para evaluar tanto el nivel de dominio que están teniendo sus estudiantes como el de sus propias acciones $\mathrm{y}$, basados en sus resultados, modificar su enseñanza; es decir, enfatizar más la evaluación formativa que la sumativa y, también, la de sus actos, para no caer en el conformismo o autocomplacencia (Bain, 2004).

Una buena docencia implica también el dominio de las habilidades comunicativas para presentar el contenido de una manera organizada, coherente y dinámica. Por ello, es conveniente que los materiales y apoyos para la enseñanza no sean confusos, ilegibles o cargados de información irrelevante, sino que resuman lo esencial del tema, tengan un adecuado balance entre texto e imágenes, sean claros, atractivos, entre otros aspectos (Hernández, 1995).

Para Morton (2009, como se citó en Friesen, 2011), un buen docente comparte su pasión y entusiasmo por su materia explicitando a los alumnos la importancia de la misma. Liga lo revisado en clase con tópicos o temas de actualidad, indaga sobre las experiencias del estudiante y las utiliza en su enseñanza. Plantea preguntas clave para señalar los puntos controversiales de un campo, los problemas no resueltos o las posturas existentes.

Ibarra (1999) encontró que los buenos docentes ordenan sus acciones en tareas claramente delimitadas, dominan los contenidos y la gestión de la clase, basan su enseñanza en el tipo de contenido por enseñar y en la lógica del campo disciplinar, son consistentes en su accionar, captan la atención de los alumnos, "saben engancharlos". Las medidas disciplinarias y del manejo del grupo se ponen al servicio de lograr la atención y para cumplir con los propósitos del tema, no son fines en sí mismos. Los profesores actúan con imparcialidad, utilizan diferentes ejemplos, presentan casos o problemas que enfrentaron. Hacen amena la clase, cumplen con las normas sin ser autoritarios o impositivos, no faltan a clase; cuidan el grado de dificultad del material, tratan con rigor y profundidad los temas enseñados, y esto lo hacen en un ambiente agradable de trabajo. 
Blanco (2009) encontró que los buenos docentes eran personas empáticas, capaces de transmitir su interés por la enseñanza, preocupados por explicar sus materias a partir de los problemas cotidianos y de actividades dialogantes.

Sintetizando las cualidades más mencionadas en las investigaciones reportadas, destacan que el maestro debe tener como prioridad el aprendizaje de sus estudiantes, y el conseguirlo es la razón de todos sus esfuerzos. Especifican claramente las metas de aprendizaje, los motivan y plantean retos, pero los van guiando y retroalimentando para lograr los propósitos establecidos, ejemplifican, demuestran lo que desean, se crea una atmósfera para el aprendizaje donde la evaluación se utiliza más para identificar el nivel real de aprendizaje que como un elemento acreditador. Además, aplican pertinentemente diferentes estrategias de enseñanza. Los resultados en el salón de clases, al usar adecuadamente los rasgos descritos anteriormente, son resumidos así por Mcber (2000):

En ellas (las clases), los alumnos saben lo que tienen que hacer y por qué tienen que hacerlo, ven la vinculación de lo que sabían previamente y se forman una idea de hacia dónde evolucionará su aprendizaje. Desean saber más. Entienden por qué un trabajo estuvo correcto y dónde necesita mejorarse en caso de que no haya sido así. Se sienten seguros y motivados debido al ambiente de respeto creado en el aula. Se apoyan unos con los otros y saben cuándo y a quién acudir si necesitan ayuda. Los maestros están continuamente atentos a lo que hacen sus alumnos. Muchas de las actividades de la clase son dirigidas por ellos, pero también hay una frecuente interacción entre el docente y sus estudiantes. Crean las máximas oportunidades para aprender y no se desperdicia el tiempo (p. 11).

Gestión de la clase.

Uno de los componentes de los aspectos didácticos es la gestión de las experiencias y actividades para crear un ambiente que estimule y favorezca el aprendizaje. Por gestión de la clase se entiende "el conjunto de reglas y 
disposiciones para crear y mantener un ambiente ordenado que favorezca tanto al educando como al aprendizaje" (Gauthier, 1997, p. 176). La gestión se incluye dentro de lo didáctico, aunque no esté directamente vinculada con la enseñanza de los contenidos académicos, pero es importante porque crea las condiciones para que lo primero se dé adecuadamente.

Mcber (2000) definió el clima de la clase como el resultado de las acciones del docente para incidir en la motivación por aprender de los alumnos; es el ambiente creado en el aula que puede ser ameno, relajado, tenso o suscitar temor (Gauthier, 1997). Un adecuado clima de clase se caracteriza por ser estimulante, cálido, placentero, equitativo, agradable, respetuoso y donde la finalidad es lograr unas buenas relaciones interpersonales para promover el aprendizaje (Carlos-Guzmán, 2006, 2014).

La relevancia de tomar en cuenta la gestión de la clase la estableció Shulman (1989) al explicar que, cuando los alumnos ingresan a la escuela, el aula es un ambiente muy complejo, impredecible, caótico, donde acontecen al mismo tiempo diferentes eventos, los cuales muchas veces no entienden y necesitan realizar una actividad doble: una, atender las demandas y tareas académicas y, la segunda, comportarse de acuerdo con las reglas sociales. E1 trabajo en el aula implica un constante intercambio entre los participantes, donde existen situaciones explícitas e implícitas que el estudiante debe desentrañar para responder adecuadamente a ellas. De hecho, una de las principales razones por las cuales realizan trabajos que no cumplen con lo solicitado es que no entendieron lo que se les pedía, ni les quedó claro el propósito de la actividad, ni tenían claridad sobre lo que debían hacer, ya sea porque no fueron aclaradas ni clarificadas o porque el estudiante, por falta de confianza y seguridad, no las externalizó y el maestro no se percató de esa situación.

Como parte de la gestión de la clase, el profesor necesita establecer buenas relaciones interpersonales para que los estudiantes le tengan confianza, sentirlo cercano, acudir con él para resolver cualquier duda sabiendo que contarán con su apoyo para resolver sus problemas académicos 
(Carlos-Guzmán, 2014). Unas buenas relaciones interpersonales evitarán muchos problemas, como son los disciplinarios o la falta de respeto, además de favorecer el aprendizaje de los alumnos. Recordar que la docencia es, en esencia, un proceso de interrelación personal que, para realizarse mejor, precisa del conocimiento y respeto mutuo del profesor y sus alumnos (Gauthier, 1997).

Toda enseñanza precisa de un orden ya que un ambiente caótico dificulta -si no es que impide- el aprendizaje; por eso son necesarias las reglas de comportamiento en el salón de clases. Se requieren también para regular y predecir los comportamientos de todos, de manera que las actividades tengan un flujo continuo y permitan aumentar las oportunidades de aprendizaje de los educandos. No es la obediencia ciega a las reglas impuesta por otros, sino la aceptación de la necesidad de cumplir con ellas en beneficio tanto propio como de todos. Ellas regulan a los alumnos y al maestro.

Un punto importante para que funcionen las reglas y dirijan el comportamiento de los involucrados es que sean claras, concretas, entendidas por todos, pero lo crucial es que su aplicación sea coherente y homogénea, no circunstancial, o existan excepciones arbitrarias, o se dejen de utilizar durante un tiempo y, luego, querer volver a hacerlo.

Entre las acciones a realizar para obtener una adecuada gestión de la clase están la estructuración clara de la tarea o actividad, un adecuado manejo del tiempo destinado al aprendizaje, organizar el espacio físico y el establecimiento de reglas de comportamiento para mantener la disciplina y el orden en la clase (Santrock, 2002; Tuckman \& Monetti, 2011).

Una de ellas es el uso de rutinas para que los alumnos poco a poco vayan entendiendo qué tipo de acciones ocurren habitualmente en el salón y, así, el docente estructure mejor su clase, de manera que ellos sepan qué hacer en cada momento. La intención, además de organizar mejor las actividades y darle fluidez a la clase, es que los estudiantes sepan lo que pasará y lo que se espera de ellos. Tal como lo establece Meirieu (2004): 
El objetivo es que el alumno esté mentalmente disponible para el trabajo que se le pedirá, que centre su atención y que, para ello, sus actividades escolares estén organizadas de forma suficientemente clara por rituales que tengan sentido (p. 220).

Las rutinas sirven igualmente para reducir la incertidumbre en el salón de clases, y permiten hacer un uso eficaz del tiempo y de las actividades escolares. Una vez establecidas, habrá el tiempo necesario para explicar en cada clase lo que se tiene que hacer. También le ayudan a supervisar adecuadamente las tareas realizadas; se ha encontrado que la rutina es una de las principales herramientas para favorecer el aprendizaje (Gauthier, 1997).

Otra acción favorable es el manejo del tiempo destinado al aprendizaje. Hay evidencias de que, mientras más tiempo pase un estudiante en actividades académicas, mejor será su rendimiento escolar, y lo opuesto sucederá si no recibe clases o el tiempo se dedica a otras actividades no académicas (Day, 1997). Entonces, lo más importante no es de cuánto tiempo dispone el profesor para su clase, sino la calidad del uso que haga al dedicarlo a las actividades académicas. A esto se le llama el tiempo comprometido en el aprendizaje (Carnoy, 2005).

De acuerdo con Gauthier (1997), los maestros que gestionan adecuadamente la clase son aquellos que supervisan el desarrollo de las actividades, son capaces de reconocer y anticipar los actos de indisciplina que pudieran contaminar al grupo y perturbar el orden. El docente cuenta con reglas para funcionar y servir a los propósitos educativos. Este autor afirma que los maestros eficaces consagran menos tiempo a administrar la clase y más a las actividades sustantivas, que son aquellas que promueven los aprendizajes.

Tuckman y Monetti (2011) resumen así la importancia del manejo adecuado por parte del docente respecto de la gestión de la clase: 
La decisión del momento y naturaleza de la intervención es una importante habilidad del profesor. Cada estudiante, salón de clase y situación son únicos; por lo tanto, intentar aplicar una perspectiva uniforme para realizar intervenciones solo producirá frustraciones. No solo es importante considerar el momento y el motivo de intervención, sino cómo lo realiza. Lo que el profesor dice y hace tiene gran poder en el salón de clase y puede lastimar los sentimientos de sus alumnos con facilidad; por lo tanto, es importante que sus intervenciones se realicen sin ira ni sarcasmo (pp. 449-450).

\section{El pensamiento, visiones y creencias del maestro.}

Con respecto a los factores que afectan tanto de manera positiva como negativa a la docencia, la investigación educativa ha encontrado el pensamiento, significados, visiones y creencias que tienen los docentes acerca de los temas propios de su profesión (Boulton-Lewis, Smith, McCrindle, Burnett \& Campbell, 2001; Goodyear \& Hativa, 2002; Hativa, 2000; Kane, Sandretto \& Heath, 2002; Monroy \& Díaz, 2001; Saroyan, 2001; Van den Berg, 2002). Lo anterior se justifica en el sentido de que, regularmente, cierto tipo de pensamiento, creencias y significados de los profesores va a producir determinada manera de enseñar y de evaluar. Por ejemplo, es muy diferente el grado de compromiso y de acciones docentes si el maestro piensa que todos sus alumnos quieren y pueden aprender (Monroy \& Díaz, 2001) y tiene altas expectativas sobre ellos (Friesen, 2011), a diferencia de otro que piensa "que la letra con sangre entra", o que tiene bajas expectativas sobre la capacidad de sus estudiantes o es poco comprometido con la docencia. Estos "marcos mentales", como los llama Hattie (2012), son fundamentales en la enseñanza.

El pensamiento docente está compuesto principalmente por su visión o aproximación a la enseñanza, los fines que cree ella tiene, sus definiciones personales sobre lo que significa el aprender y cómo entiende la evaluación.

Por lo anterior, hay que identificar las visiones de los maestros sobre la enseñanza, ya que determinan los métodos de enseñanza utilizados, el tipo de 
tareas planteadas a los estudiantes, las razones por las cuales hacen lo anterior y las formas elegidas de evaluación, así como su grado de compromiso con la labor docente (Carlos-Guzmán 2006, 2014).

El aprendizaje de los estudiantes se verá poco favorecido si el docente piensa que su función es transferir su conocimiento disciplinario a la mente de sus alumnos (Kember \& Gow 1994, como se citó en Weimer, 2002). O, como dice Friesen (2011), pensar que su labor es, únicamente, traspasar las notas de clase a los apuntes de los alumnos. Por ello, lograr un aprendizaje profundo o la comprensión por parte de los estudiantes será más probable de suceder si el docente tiene una teoría de la enseñanza compleja, sofisticada y profunda. También, la práctica docente será poco efectiva si piensa que tiene poca capacidad en influir en el aprendizaje de sus alumnos, como ampliaremos en el siguiente apartado.

Hay diferentes modelos de enseñanza de los maestros (Ramsden, 2007; Goodyear \& Hativa, 2002; Entwisle \& Walter, 2002; Martin, Prosser, Trigwell, Ramsden \& Benjamín, 2002; Kember \& Por-Kwam, 2002). Uno de los más citados es el de Biggs y Tang (2009), quienes identifican tres tipos: 1) Transmitir información cuyo ejemplo es la enseñanza tradicional y dar gran peso a comunicar lo disciplinario; 2) el centrado en el maestro; en este caso, la prioridad son las acciones del docente: la responsabilidad del aprendizaje descansa en él y no en lo que el alumno hace, y 3) el centrado en el aprendizaje, donde se requiere que el estudiante sea un activo participante. En este tercero, lo importante son las acciones de los alumnos, no tanto las del maestro; donde lo relevante es ponerlo en contacto con el objeto por aprender. Por ello, la labor del docente consiste en asesorarlo y corregirlo o ayudarlo a encontrar la solución a los problemas planteados o completar adecuadamente las tareas académicas. Como menciona Resnick (1981), "la enseñanza no debe diseñarse para introducir conocimientos en las cabezas de los estudiantes, sino para poner a los alumnos en situaciones que les permitan construir un conocimiento bien estructurado" (p. 660). 
Llegar a este nivel implica para el maestro confrontar las visiones con las que fue formado, como creer que el centro de todo el acto de enseñanza es lo que él hace o lo único importante es lo disciplinario. En cambio, un docente con una visión centrada en el aprendizaje actuaría como lo describe Meirieu (2005):

(En la enseñanza) ya no cabe preguntarse “¿Qué les voy a decir?”, sino “¿Qué les voy a pedir que hagan?”. Por supuesto, sin renunciar a la clase magisterial cuando sea necesario, pero preguntándose una vez más “¿Cómo pueden mis alumnos sacar el máximo provecho de la clase?”, “qué consejos pueden captar su atención?”, “¿qué ejercicios puedo mandarles a hacer para comprobar de manera continuada que mis consejos son los adecuados?” (p. 56).

\section{Motivación, responsabilidad y autoeficacia docente.}

Las últimas dimensiones que también afectan el desempeño del maestro son los aspectos docentes, como su motivación y compromiso, así como sentirse responsable por el aprendizaje de sus alumnos (Carlos-Guzmán, 2006; Goodyear \& Hativa, 2002; Hativa, 2000; Hernández, 1995). El disfrutar la docencia es una cualidad que tienen los buenos docentes (Carlos-Guzmán, 2006, 2014).

Desde los 90 surgió con gran fuerza el estudio sobre la autoeficacia del maestro referido a la confianza para resolver los problemas de su docencia, al descubrirse en numerosas investigaciones que era un componente crucial de su calidad; así, por ejemplo, Tschannen, Woolfook \& Hoy (1998) definieron la eficacia docente como "el grado en que un maestro cree que tiene la capacidad de afectar la ejecución del estudiante". Otros autores, como Guskey y Passaro (1994, como se citó en Tschannen, Woolfook \& Hoy, 1998), la conceptualizan así: “(es la) convicción (del docente) de que puede influir en qué tan bien aprenden los estudiantes a pesar de tener alumnos difíciles o poco motivados". Rotter (1966) estableció que un maestro actuará mejor si piensa que tiene la capacidad para controlar o afectar el rendimiento 
académico y motivación del estudiante, más que los factores contextuales o externos a la escuela.

La autoeficacia docente implica el sentirse responsable por el aprendizaje de sus estudiantes, no culparlos por los fracasos académicos; mantenerse motivado en lo que hace pese a las adversidades, limitaciones o dificultades propias de su labor.

Tschannen, Woolfook y Hoy (1998) señalan que las investigaciones demuestran la importancia de la autoeficacia docente, ya que los maestros con alto sentido de autoeficacia están abiertos a probar otras formas de enseñanza, muestran un alto nivel de planeación y organización, son más tolerantes con los errores de sus estudiantes y están más dispuestos que otros a trabajar con alumnos deficientes. Asimismo, enseñan con gran entusiasmo y es poco probable que abandonen la docencia. A nivel de escuela, un profesor con estas características contribuye a mejorar el clima laboral, influye para que la toma de decisiones se centre en lo académico, así como a mejorar el trabajo colegiado de los docentes.

Ahora, siendo tan importante, ¿cómo se puede formar o desarrollar la autoeficacia?, ya que es afectada, tanto positiva como negativamente, por los factores escolares como son el clima laboral, el apoyo del director y la forma en que se toman las decisiones, entre otros. En este caso, es crucial que el maestro sienta que tiene la libertad de tomar decisiones académicas; en cambio, le afectan negativamente un mal ambiente laboral, los salarios bajos, la falta de reconocimiento, las excesivas demandas y el aislamiento. Por eso es muy importante el apoyo y la colaboración de los colegas para hacer un trabajo colegiado estimulante.

Los factores que ayudan a la autoeficacia del profesor son la autopercepción de sus competencias docentes, de sentirse capaz de identificar los requerimientos de una situación particular de enseñanza y creer que cuenta con las herramientas requeridas para resolver adecuadamente un problema de enseñanza. Tiene una naturaleza cíclica o autorreforzante; el tener éxito en una tarea contribuye a enfrentar mejor otra parecida. Los logros pasados 
otorgan confianza y son una fuente para encarar adecuadamente futuras demandas. Mediante este tipo de experiencias, el maestro desarrolla un conjunto de creencias cruciales sobre sus habilidades.

Algunas de las estrategias para formar la autoeficacia docente son contar con un facilitador que centre la atención a la calidad docente, realizar actividades que fomenten la autoevaluación y la reflexión (Arbesú \& Argumedo, 2010), ayudarlos a analizar la tarea, descomponerla y recibir apoyo para modificar su práctica docente. Otra manera es cuestionar los supuestos o el pensamiento didáctico que tiene el maestro sobre la enseñanza porque, si no hay una reformulación de su papel como docente, difícilmente modificará sus formas de enseñanza. Cuando se está trabajando para lograrlo, es muy importante centrarse en sus logros y avances, en fortalecer el locus interno o la sensación de que ellos pueden afectar positivamente el rendimiento de los estudiantes más que los factores externos a la enseñanza; es decir, es crucial darles un sentido de control y responsabilidad en lo que hacen.

Finalmente, los autores señalan que se necesita estudiar más los factores que facilitan o inhiben el desarrollo de las creencias eficaces. Igualmente, se requiere investigar cómo los maestros se empeñan en desear enseñar bien a pesar de las dificultades que enfrentan.

\section{Dimensiones para evaluar la labor docente. Una propuesta.}

En esta segunda parte, y luego de haber analizado los aspectos cruciales de la docencia, se presenta la propuesta para evaluar al maestro dividida en dos categorías: una referida a los instrumentos sugeridos para ello o el cómo hacerlo, y la segunda ofrece un desglose de qué evaluar, profundizando en los aspectos didácticos, al ser el núcleo central de lo propuesto y que pugnamos sean los prioritarios para evaluar al docente.

Proponemos que las cuatro dimensiones antes descritas requieren ser evaluadas de manera diferencial; es decir, utilizando distintos instrumentos para hacerlo y no solamente uno. En la Tabla 1 aparece la relación entre la dimensión de la enseñanza evaluada y la sugerencia de formas para hacerlo. 


\section{Tabla 1.}

Formas de evaluar las dimensiones de la docencia.

\begin{tabular}{ll}
\hline Dimensión evaluativa & Forma de evaluación \\
\hline 1. Dominio disciplinar & $\begin{array}{l}\text { Examen y/o ensayo sobre el dominio del conocimiento } \\
\text { de las materias o grados que imparte, así como de su } \\
\text { programa académico. }\end{array}$ \\
& $\begin{array}{l}\text { Diseño de una planeación didáctica. } \\
\text { La observación por un experto de la práctica docente. } \\
\text { Evaluación a los alumnos sobre los contenidos } \\
\text { enseñados, o las viñetas, estudio de casos y } \\
\text { simuladores. }\end{array}$ \\
2. Los aspectos didácticos & $\begin{array}{l}\text { Cuestionarion } \\
\text { la docencia, presentación de casos o viñetas. }\end{array}$ \\
3. Pensamiento didáctico & Cuestionario para maestros, viñetas y casos. \\
4. Motivación, autoeficacia y &
\end{tabular}

Lo anterior se refiere a cómo evaluar el desempeño docente; ahora, para ilustrar el qué evaluar, nos centraremos en demostrar la manera de valorar la dimensión didáctica: parte esencial de nuestra propuesta y por su grado de generalidad para evaluar el desempeño docente en diferentes niveles educativos.

Pensamos que la mejor manera de evaluar este aspecto es observando el desempeño del docente en el aula y no mediante un cuestionario, ya que ambos evalúan aspectos distintos: el primero mide el desempeño y, el segundo, las opiniones de los receptores del acto docente. Para apoyar esta opción se cita lo expresado por Schmelkes (2014):

Es en la práctica donde la planeación se pone a prueba, donde el docente pone a prueba su capacidad de enfrentar imprevistos, donde su preocupación por el aprendizaje de todos sus alumnos cobra o no visibilidad, y donde el verdadero oficio de ser docente se manifiesta... los resultados del aprendizaje son consecuencia de diversos procesos, y el más importante de ellos es el de la enseñanza (p. 158).

1 Si bien hemos sido críticos con el uso de los cuestionarios en la evaluación docente, no los descartamos dado que tienen su utilidad si son empleados adecuadamente para valorar los aspectos donde pueden hacerlo, como son el pensamiento didáctico, la motivación, la autoeficacia y la responsabilidad docente. 
Por eso, la observación del manejo didáctico del maestro tendría que ser evaluada por un experto en evaluación docente y/o por un maestro con amplía experiencia, al requerirse su juicio experto para juzgar si lo realizado es adecuado o no, si es pertinente para alcanzar los fines de la enseñanza, entender el momento en que se encuentra la clase observada y justificar su valoración. Así, la evaluación será mucho más que solo verificar si está presente o ausente determinado atributo. Para fines prácticos de la propuesta sugerimos que ella se videograbe, a fin de que este desempeño pueda ser valorado por un grupo de expertos que no necesitarían estar presentes en el salón de clases; además, se tendrá un producto permanente que podrá revisarse las veces que sea necesario. De proceder así se evitarán muchos problemas y se reducirán costos de la evaluación.

Con respecto a las tres dimensiones restantes, solo ofrecemos algunas directrices para evaluarlas, aceptando que se precisaría ampliarlas para diseñar instrumentos ex profeso adecuados a la materia o disciplina, al nivel educativo y al tipo de institución.

Sugerimos que la observación tendría que tomar una muestra de una clase y conducirse éticamente. Se aclara que lo propuesto no es una lista cotejable porque no se trata de realizar todas, sino solo aquellas pertinentes y adecuadas para el momento del curso que se observa; eso es lo que el experto juzgaría. Por eso, se dan ejemplos de diversas acciones que pueden valorarse considerando las variadas formas que asume la docencia. 


\section{Tabla 2.}

\section{Aspectos a evaluar de las dimensiones del acto docente.}

\begin{tabular}{ll}
\hline Dimensiones & Aspectos a evaluar \\
\hline 1. Dominio disciplinar & $\begin{array}{l}\text { Demostrar el adecuado manejo de los contenidos que enseña, su } \\
\text { programa y grado. }\end{array}$ \\
2. Dominio didáctico y de & Las acciones aplicadas buscan el aprendizaje del estudiante. \\
gestión & Demuestra haber preparado y planeado la clase. \\
& La clase es organizada; hay fases claramente delimitadas, como inicio, \\
& desarrollo y cierre. \\
& La estrategia didáctica utilizada es idónea para alcanzar los propósitos \\
& del tema por enseñar, adaptada al tipo de estudiantes y contenido, así \\
& como al contexto escolar.
\end{tabular}

Inicio

Identifica las ideas y conocimientos previos de los estudiantes. Hace un diagnóstico del nivel en que se encuentran.

Comunica los propósitos de enseñanza y los productos que solicitará para valorar si ellos fueron alcanzados. Clarifica o resalta la importancia del tema, despertó el interés (vgr. estableció los propósitos de la clase, planteó retos intelectuales, o formuló un problema o realizó un experimento e hizo preguntas sobre lo observado, o hizo alusión a una situación de la vida diaria, recordó, repasó lo visto en la clase anterior, etc.).

\subsection{Dominio didáctico}

Contextualizó y ubicó lo que se revisará, lo vinculó con otros temas y contenidos.

O realizó alguna dinámica o ejercicio para introducir su tema.

\section{Desarrollo}

Enseñó claramente. Realizó varias de estas acciones.

Simplificó el material, explicó siguiendo un orden lógico.

Utilizó un lenguaje sencillo y comprensible para los alumnos. Desglosó el tema en pasos pequeños. Resaltó los puntos importantes. Adaptó su enseñanza al nivel de conocimientos y características de los alumnos. Puso ejemplos para ilustrar los conceptos empleados. No redundó en información innecesaria.

Retroalimentó de una manera clara y amable las realizaciones de los estudiantes. Lo hizo de manera constructiva y dio sugerencias concretas de mejora. 
Su ritmo de enseñanza no es ni demasiado lento ni muy rápido.

Verifica que los estudiantes estén comprendiendo lo expuesto.

Utiliza el "barómetro" o checa el comportamiento verbal y no verbal de sus estudiantes y, con base en ello, hace cambios inmediatos o mantiene lo que hace.

Hace una "construcción conjunta" del conocimiento por medio de un diálogo con los alumnos donde plantea preguntas o situaciones problema, pide su participación, va corrigiendo lo que dicen los estudiantes y da pautas hasta llegar a una síntesis o definición del concepto o principio.

Habilidades comunicativas. Ejecuta varios de estos comportamientos.

Su tono de voz es audible para todos los estudiantes, pausada, con matices, enfatiza lo importante, no es plana; hay mínimas "muletillas".

Se desplaza al exponer, tiene contacto visual con sus alumnos, utiliza el lenguaje corporal para comunicar el tema y muestra entusiasmo.

Hace un manejo adecuado del pizarrón, de las presentaciones y de los materiales

Su letra es clara, de buen tamaño y entendible.

Si utiliza presentaciones son resúmenes de lo más importante, su formato es atractivo y comprensible.

Explica las diapositivas, no las lee.

\section{Afianza la comprensión del nuevo tema}

Modela las aplicaciones de lo expuesto o les da ejemplos del tipo de trabajo o producto solicitado.

O utiliza una rúbrica para clarificar cómo debe ser el trabajo pedido.

Los estudiantes tienen la oportunidad de practicar o ejercitar lo enseñado como, por ejemplo, solicitarles que lo apliquen en un caso, problema o ejercicios o pide ejemplos originales.

Utiliza pertinentemente el aprendizaje cooperativo. Promueve así tanto el dominio de los contenidos como el desarrollo de habilidades psicosociales y de cooperación.

Luego de exponer el tema hace un resumen de los puntos más importantes. Resalta las implicaciones, ventajas o limitaciones del tema tratado. $\mathrm{O}$ pide a los alumnos que resuman lo revisado 


\section{Evaluación}

\subsection{Gestión de la clase}

Empleo de materiales didácticos o de TIC de manera adecuada a los fines de la clase y al tipo de alumno.

Si aplica otro tipo de estrategia didáctica como el estudio de caso, la solución de problemas o por proyecto. Son pertinentes para el tema, los propósitos de la enseñanza y el nivel de los alumnos.

Las emplea adecuadamente siguiendo los pasos y fases estipulados en cada una de las estrategias.

Cierre

Utiliza el instrumento adecuado para el tipo de conocimiento evaluado (declarativo, procedimental y actitudinal).

Los instrumentos de evaluación están diseñados correctamente.

Emplea la evaluación primordialmente para retroalimentar. Los estudiantes saben por qué sus respuestas estuvieron acertadas o equivocadas.

Les informa continuamente a los estudiantes sobre su desempeño académico.

Califica de manera justa y adecuada.

Establecimiento de las tareas o actividades describiendo por escrito los propósitos y las instrucciones, proporcionó los materiales para hacerla. Comunico cómo y cuándo serán evaluados. Dio a conocer oportunamente los criterios y requisitos para evaluar.

Las tareas estimulan o favorecen los procesos cognoscitivos complejos, no son simples o memorísticas.

Hay reglas en el salón de clases para una adecuada convivencia: se aplican tanto a docentes como a los estudiantes.

Uso de rutinas.

Manejo adecuado de los tiempos y ritmos de la clase.

La mayor parte del tiempo de la clase está dedicado a las actividades académicas.

Hay orden y organización.

Fluidez de la clase, están claramente delimitadas las actividades, no se traslapan.

Espacio físico adecuado a los propósitos de la enseñanza, le permite monitorear continuamente el comportamiento de los alumnos en el salón de clases. 
2.2. Gestión de la clase

Evaluación global del desempeño docente

Pensamiento didáctico

Autoeficacia, motivación y responsabilidad docente
Crea un ambiente que estimule y propicie el aprendizaje. Hay respeto, confianza y los alumnos se encuentran a gusto.

Interacción personal adecuada y respetuosa.

Evaluar integralmente el desempeño docente y justificar la decisión.

Recomendaciones y sugerencias:

¿Qué es para usted enseñar?

¿Cuáles son los fines de su enseñanza?

¿Qué es el aprendizaje para usted?

¿Qué es la evaluación y para qué evalúa?

¿Qué cualidades tiene un buen docente?

¿Cómo sabe que sus alumnos aprendieron?

¿Cuáles son las acciones más importantes que hace para estimular el aprendizaje de los alumnos?

Mencione las causas o razones por las cuales es maestro.

¿Qué aspectos considera gratificantes de su trabajo y cuáles no? ¿Por qué?

Si sus alumnos no aprenden, ¿cuál cree que es la principal causa?

Describa un problema que haya tenido en su práctica docente, indique cómo lo resolvió y, si lo hizo, a qué lo atribuye.

\section{Conclusiones}

Ofrecimos una alternativa a la manera usual de evaluar el desempeño docente, para lo cual identificamos, de los resultados de la investigación educativa, cuatro dimensiones cruciales del acto de enseñar, y para cada una de ellas proporcionamos sugerencias sobre cómo valorarlas. Consideramos que las ventajas de lo propuesto son: 1) Basarse en lo que sabemos sobre los mecanismos que apoyan un mejor aprendizaje e incrementan el rendimiento académico de los estudiantes; por eso describimos los resultados de la investigación educativa que justifican lo propuesto; 2) Ofrecer criterios claros para una valoración justa e integral de la práctica docente, al tomar en cuenta lo sustantivo de ella y no lo superfluo o innecesario; 3) Enfatizar la evaluación formativa, de manera tal que el maestro pueda identificar sus puntos fuertes 
y débiles para enseñar mejor y utilizarlos para diseñar rutas de formación y actualización docente ajustadas y pertinentes para el contexto donde realiza su labor; 4) Al haber claridad de lo que se evalúa del desempeño docente, se favorece que estos procesos sean justos, rigurosos y transparentes; así, las decisiones tomadas sobre el desempeño docente serán fundamentadas y centradas en lo académico y menos sesgadas, arbitrarias, limitadas o simplistas. Tomar en cuenta estos factores tiene mayor valor cuando, basados en los resultados de la evaluación docente, se toman decisiones para premios y reconocimientos, así como para el ingreso, permanencia o promoción del docente.

Expusimos que la docencia es una actividad compleja, multidimensional y contextualizada. Donde hay la polémica de si es una actividad que muestra rasgos generalizados que emplearía un docente independientemente del nivel educativo que enseña o si, por el contrario, es una acción específica y diferente dependiendo del contexto o del nivel educativo donde se imparta (Gauthier, 1997). Nosotros nos adscribimos a la propuesta generalista y, si bien reconocemos que en cada nivel educativo hay rasgos particulares, pensamos que existen más elementos compartidos que específicos, como son los aspectos didácticos que todo maestro debe dominar independientemente del nivel educativo. De hecho, varias de las investigaciones presentadas aquí fueron obtenidas de investigaciones realizadas en diferentes niveles educativos, asumiendo que lo didáctico es lo crucial para el desempeño docente y la razón para describir con mayor detalle cómo podría ser evaluado.

Nuestra propuesta da prioridad al juicio evaluativo del desempeño docente y menos a los resultados de los cuestionarios evaluativos, ya que establecimos que enseñar es un procedimiento de tipo heurístico donde existen varias opciones ante una situación, de ahí que se requiera valorar lo que haría el maestro ante una determinada situación y observar los efectos de sus acciones en la clase. Por eso le damos tanta importancia al juicio experto y a reclutar personas con amplia experiencia tanto en la docencia como en la evaluación. 
A diferencia de los instrumentos tradicionales, las acciones propuestas no tendrían que realizarse todas, sino observar si son utilizadas las pertinentes para los propósitos de la enseñanza, las características de los estudiantes, el tipo de contenidos, el momento y la situación didáctica. Esta es la justificación de proponer que el maestro debe ser evaluado observando su desempeño y juzgado por expertos que determinen si lo que hace es adecuado o no.

Al tener tanto peso los resultados de la evaluación docente, hay que ser sumamente cuidadoso para que este proceso sea hecho con el mayor rigor y transparencia posibles, para evitar su distorsión apareciendo comportamientos poco éticos, ilegales o incluso de intimidación hacia los evaluadores. Eso justifica disponer de un producto permanente como es la filmación de la clase.

Pensamos que esta sería la evaluación ideal; pero, si trata de evaluar a un gran número de docentes, lo aquí propuesto sobre los aspectos didácticos podría emplearse para valorar el comportamiento del maestro ante una situación didáctica, mediante estudios de caso o simuladores, que bien trabajados serían más realistas y adecuados que un examen de conocimientos, como a veces son valorados los profesores.

Con respecto a las otras tres dimensiones, ofrecimos algunos lineamientos porque, en su concreción, tendría que ser particular y ajustada al nivel educativo, disciplina y tipo de institución. Lo crucial es que todos los instrumentos sugeridos se completen y permitan valorar integral, adecuada y justamente algo tan complejo como es el desempeño docente. Como dijimos al inicio, lo obtenido debería de emplearse para retroalimentar, corregir el desempeño del maestro y ayudarlo a enseñar mejor.

Hacer de la evaluación docente un proceso que dé prioridad a lo formativo más que a lo punitivo requiere fomentar, a nivel institucional, una cultura de evaluación (Rueda, Luna, García \& Loredo, 2010), de rendición de cuentas y de trasparencia de lo hecho en las escuelas, donde lo prioritario sean los aspectos académicos, desear el mejoramiento de la calidad educativa y no 
los de otro tipo. Sin olvidar que el sentido de todo este proceso es mejorar el desempeño de los docentes para apoyarlos a hacer cada vez mejor ese acto tan complejo y difícil como es lograr que otro ser humano aprenda.

\section{Referencias}

Andreucci, P. (2011). La dimensión interaccional en la supervisión de prácticas docentes: una aproximación desde la transdisciplinariedad. Revista chilena Calidad en Educación, 34, 185-202. Recuperado de http://www.scielo.cl/pdf/caledu/n34/art10.pdf

Arbesú, M., \& Argumedo, G. (2010). El uso del portafolio como recurso para evaluar la docencia universitaria. Revista Iberoamericana de Evaluación Educativa, 3(1). Recuperado de http://www.rinace.net/riee/numeros/ vol3-num1_e/art10.pdf

Assaél, J., \& Pavez, J. (2008). La construcción e implementación del sistema de evaluación docente chileno: principales tensiones y desafíos. Revista Iberoamericana de Evaluación Educativa, 1(2). Recuperado de https:// dialnet.unirioja.es/servlet/articulo?codigo $=2789094$

Bain, K. (2004). What the best college teachers do. USA: Harvard University Press.

Biggs, J., \& Tang, C. (2009). Teaching for quality learning at University. United Kingdom: McGraw-Hill.

Blanco, A. (2009). Aprender a enseñar. Barcelona: Paidós.

Boulton-Lewis, G., Smith, A., McCrindle, A., Burnett, P., \& Campbell, K. (2001). Secondary teacher's conceptions of teaching and learning. Learning and instruction, 11, 35-51. http://dx.doi.org/10.1016/S09594752(00)00014-1

Butsch. R. (1931). Teacher rating. Review of Educational Research, 1(2), 99107. http://dx.doi.org/10.2307/1168127

Calderón, A., \& de Oliveira, R. (2013). Evaluación por resultados y la cultura de la "performatividad": La evaluación docente en la escuela pública del Estado de San Pablo (Brasil). Revista Iberoamericana de Evaluación Educativa, 7(9). 43-52. Recuperado de http://www.rinace. net/riee/numeros/vol7-num2e/art2.pdf 
Carlos-Guzmán, J. (2006). El pensamiento didáctico de los profesores considerados como buenos por los alumnos de la Facultad de Psicología de la UNAM (Tesis de maestría inédita). Universidad Nacional Autónoma de México, México.

Carlos-Guzmán, J. (2014). Las buenas prácticas de enseñanza de los profesores de la Facultad de Ingeniería de la UNAM (Tesis de doctorado inédita). Universidad Nacional Autónoma de México, México.

Carnoy, M. (2005). Mejorando la calidad de la educación ¿Qué sirve? En Conferencias magistrales del VIII Congreso Nacional de Investigación Educativa. México: COMIE, 79-88.

Cisneros, E., Jorquera, M., \& Aguilar, A. (2011). Validación de instrumentos de evaluación docente en el contexto de una universidad española. Revista Iberoamericana de Evaluación Educativa, 3(1). Recuperado de http:// riied.uniandes.edu.co/archivos/Taller_1/EdithCisneros_MariaJorquera_ AngelAguilar.pdf

Cordero, G. (2002). Consideraciones generales sobre el uso del portafolio de desempeño docente en educación superior. Revista Acción Pedagógica, 11(2), 76-83. Recuperado de http://dialnet.unirioja.es/servlet/ articulo? codigo $=2973011$

Darling-Hammond, L. (2000). Teacher quality and student achievement. A review of state policy evidence. Education Policy Analysis Archives, 8(1). http://dx.doi.org/10.14507/epaa.v8n1.2000

Darling-Hammond, L. \& Youngs, P. (2002). Defining "Highly Qualified Teachers: what does "scientifically-based research" actually tell us? Educational Researcher. 31(9), 13-25. http://dx.doi. org/10.3102/0013189x031009013

Day, C. (1997). Pasión por enseñar. La identidad personal y profesional del docente y sus valores. España: Narcea.

Dewey, J. (2006). Democracia y educación. Madrid: Morata.

Echeverría, R. (2011). Escritos sobre aprendizaje: Recopilación. Buenos Aires: Granica.

Entwistle, N., \& Walter, P. (2002). Strategic alertness and expanded awareness within sophisticated conception of teaching. In P. Goodyear y N. Hativa 
(Eds.) Teacher thinking, beliefs and knowledge in higher education. USA: Kluwer Academic Publisher. http://dx.doi.org/10.1007/978-94010-0593-7_2

Friesen, N. (2011). The lecture as a trans medial pedagogical form: a historical analysis. Educational Researcher, 40(3), 95-102. http://dx.doi. org/10.3102/0013189X11404603

García, J., \& Medécigo, A. (2014). Los criterios que emplean los estudiantes universitarios para evaluar la ineficacia docente de sus profesores. Perfiles Educativos, 26(143). Recuperado de http://www.revistas.unam. mx/index.php/perfiles/article/view/44026/39835

Gauthier, C. (1997). Pour une théorie de la pédagogie. Recherches contemporaines sur le savoir des enseignants. Francia: De Boeck Université.

Goodyear, P., \& Hativa, N. (2002). Introduction. In P. Goodyear y N. Hativa (Edits.), Teacher thinking, beliefs and knowledge in higher education. USA: Kluwer Academic Publishers. http://dx.doi.org/10.1007/978-94010-0593-7_1

Hativa, N. (2000). Teaching for effective learning in Higher Education. Dordrecht/Boston/London: Kluwer Academic Publishers. http://dx.doi. org/10.1007/978-94-010-0902-7

Hattie, J. (2009). Visible learning. A synthesis of over 800 meta-analyse relating achievement. London \& New York: Routledge, Taylor \& Francis Group.

Hattie, J. (2012). Visible learning for teachers. Maximizing impact on learning. London \& New York: Routledge, Taylor \& Francis Group.

Hernández, P. (1995). Diseñary enseñar. Teoría y técnicas de la programación $y$ del proyecto docente. Madrid: Narcea.

Huba, M., \& Fred, J. (2000). Learner-center assessment on college campuses. Shifting the focus from teaching to learning. USA: Allyn\& Bacon.

Ibarra, L. (1999). La educación universitaria y el buen maestro. México. Gernika.

Jornet, J., González, J., \& Bakieva, M. (2012). Los resultados de aprendizaje como indicador para la evaluación de la calidad de la docencia 
universitaria. Reflexiones metodológicas. Revista Iberoamericana de Evaluación Educativa, 5(2). Recuperado de http://www.rinace.net/riee/ numeros/vol5-num2/art7.html

Kane, R., Sandretto, S., \& Heath, C. (2002). Telling half of the story: a critical review of research on the teaching beliefs and practices of university academics. Review of Educational Research, 72(2), 177-228. http:// dx.doi.org/10.3102/00346543072002177

Kember, D., \& Por-Kwam., K. (2002). Lecturer's approaches to teaching and their relationship to conception of good teaching. En P. Goodyear $\&$ N. Hativa (Eds.), Teacher thinking, beliefs and knowledge in higher education. USA: Kluwer Academic Publishers.

Loera, J. \& Cazares, L. (2005). Las perspectivas de los alumnos sobre las escuelas PEC. Reporte de las primeras 4 fases de los cuestionarios de factores asociados al aprovechamiento escolar. Chihuahua: Heurística Educativa.

Martin, E., Prosser, M., Trigwell, K., Ramsden, P., \& Benjamín, J. (2002). What university teacher teach and how they teach it? En P. Goodyear y N. Hativa (Eds.), Teacher thinking, beliefs and knowledge in higher education. USA: Kluwer Academic Publishers. http://dx.doi. org/10.1007/978-94-010-0593-7_6

Martínez Rizo, F. (2011). La evaluación del profesorado. Conferencia en las VII Jornadas de Cooperación Educativa sobre Evaluación.

Mcber, H. (2000). Research into teacher effectiveness. A model of teacher effectiveness. Research report $\mathrm{N}^{0}$ 216. Department for Education and Employment. United Kindom. Recuperado de http://dera.ioe. ac.uk/4566/1/RR216.pdf

Meirieu, P. (2004). En la escuela hoy. Barcelona: Octaedro.

Meirieu, P. (2005). Carta a un joven profesor. ¿Por qué enseñar hoy? Barcelona: Graó.

Monroy, M., \& Díaz, M. (2001) La evaluación de los docentes a través de la reflexión de las teorías y creencias de los docentes. En: M. Rueda, F. Díaz Barriga y M. Díaz (Comps.) Evaluar para comprender y mejorar la docencia en educación superior, pp. 125-138. México: UAM-UNAMY UABJO. 
Nathan, M., \& Petrosino, A. (2003). Expert blind spot among preservice teachers. American Educational Research Journal, 40(4), 905-928. http://dx.doi.org/10.3102/00028312040004905

Onrubia, J., Rochera, J., \& Colomina, R. (2001). Interactividad, mecanismos de influencia educativa y construcción del conocimiento en el aula. En: C. Coll, J. Palacios y A. Marchesi. Desarrollo Psicológico y educación 2. Psicología de la educación escolar. Madrid: Alianza Editorial.

Organización para la Cooperación y el Desarrollo Económico (2011). La medición del aprendizaje de los alumnos. Mejores prácticas para evaluar el valor agregado de las escuelas. Francia: OCDE.

Patrón, R., \& Cisneros, E. (2013). Los sistemas de estímulos académicos y la evaluación de la docencia: experiencia de dos universidades. Revista Iberoamericana de Evaluación Educativa, 7(9), 85-95. Recuperado de http:/www.rinace.net/riee/numeros/vol7-num2e/art6.html

Posner, G. (2005). Analizando el currículum. México: MacGraw-Hill.

Ramsden, P. (2007). Learning to teach in higher education. London and New York: Routledge Falmer.

Resnick, L. (1981). Instructional psychology. Annual Review of Psychology, 23. http://dx.doi.org/10.1146/annurev.ps.32.020181.003303

Rotter, J. (1966). Generalized expectancies for internal versus external control of reinforcement. Psychological Monographs, 80, 1-28. http:// dx.doi.org/10.1037/h0092976

Rueda, M. (2001). Una propuesta de cuestionario dirigido a los estudiantes para evaluar la función docente en la universidad. En: M. Rueda, F. Díaz Barriga \& M. Díaz (Comps.). Evaluar para comprender y mejorar la docencia en educación superior (pp. 125-138). México: UAM-UNAMY UABJO.

Rueda, M., Luna, E., García, B., \& Loredo, J. (2010). La evaluación de la docencia en las universidades públicas mexicanas: Un diagnóstico para su comprensión y mejora. Revista Iberoamericana de Evaluación Educativa, 3(1). Recuperado de https://repositorio.uam.es/handle/10486/661585

Santrock, J. (2002). Psicología de la Educación. México: McGraw-Hill. 
Saroyan, A. (2001). Evaluation of teaching for improvement: a myth or a reality? En: M. Rueda, F. Díaz Barriga \& M. Díaz (Comps.). Evaluar para comprender y mejorar la docencia en educación superior (pp. 125138). México: UAM-UNAMY UABJO.

Schmelkes, S. (2014). La evaluación del desempeño docente: estado de la cuestión. En UNESCO. Temas críticos para formular nuevas políticas docentes en América Latina y el Caribe. El debate actual. París: Centro de Estudios de Políticas y Prácticas en Educación (CEPPE). UNESCO, pp. 154-186. Recuperado de http://snte.org.mx/seccion56/vernoticias. php? artids $=304 \&$ cat $=32$

Shulman, L. (1989). Paradigmas y programas de investigación en el estudio de la enseñanza: Una perspectiva contemporánea. En M. Wittrock. $L a$ investigación de la enseñanza, I. Enfoques, teorías y métodos. España: Paidós Educador.

Shute, V.(2008).Focusonformativefeedback.ReviewofEducationalResearch. March, 78(1), 153-189. http://dx.doi.org/10.3102/0034654307313795

Tschannen, M., Woolfook, A., \& Hoy W. (1998). Teacher efficacy; Its meaning and measure. Review of Educational Research, 68(2). http:// dx.doi.org/10.3102/00346543068002202

Tuckman, B., \& Monetti, D. (2011). Psicología Educativa. México: CENGAGE Learning.

UNESCO (2014). Temas críticos para formular nuevas politicas docentes en América Latina y el Caribe. El debate actual. París: Centro de Estudios de Políticas y Prácticas en Educación (CEPPE). UNESCO.

Van den Berg (2002). Teacher's meanings regarding educational practice. Review of Educational Research, 72(4), 577-625. http://dx.doi. org/10.3102/00346543072004577

Weimer, M. (2002). Learner-centered teaching. Five key changes to practice. U.S.A.: Jossey-Bass.

Zabala, A. (2000). La práctica educativa. Cómo enseñar. Barcelona: Graó. 